\title{
Impact of Fatty Liver on Acute Pancreatitis Severity
}

\author{
Seung Bae Yoon, ${ }^{1,2}$ In Seok Lee, ${ }^{1,2}$ Moon Hyung Choi, ${ }^{2,3}$ Kyungjin Lee, ${ }^{1}$ Hyoju Ham, ${ }^{1}$ \\ Hyun Jin Oh, ${ }^{4}$ Se Hwan Park, ${ }^{1}$ Chul-Hyun Lim, ${ }^{1}$ and Myung-Gyu Choi ${ }^{1}$
}

\author{
${ }^{1}$ Division of Gastroenterology, Department of Internal Medicine, College of Medicine, The Catholic University of Korea, \\ Seoul, Republic of Korea \\ ${ }^{2}$ Cancer Research Institute, College of Medicine, The Catholic University of Korea, Seoul, Republic of Korea \\ ${ }^{3}$ Department of Radiology, College of Medicine, The Catholic University of Korea, Seoul, Republic of Korea \\ ${ }^{4}$ Center for Cancer Prevention and Detection, National Cancer Center, Goyang-si, Republic of Korea
}

Correspondence should be addressed to In Seok Lee; isle@catholic.ac.kr

Received 24 December 2016; Accepted 22 March 2017; Published 27 April 2017

Academic Editor: Alessandro Zerbi

Copyright @ 2017 Seung Bae Yoon et al. This is an open access article distributed under the Creative Commons Attribution License, which permits unrestricted use, distribution, and reproduction in any medium, provided the original work is properly cited.

\begin{abstract}
Aim. Acute pancreatitis is typically a mild disease, but some patients develop severe courses. Fatty liver changes are seen in patients with acute pancreatitis, but its clinical significance has not been well-studied. We aimed to investigate the relationship between fatty liver and the severity of acute pancreatitis. Methods. Unenhanced CT images of patients with acute pancreatitis were retrospectively reviewed by a radiologist, and mean hepatic and splenic attenuation was measured in Hounsfield units (HU). Fatty liver was defined as mean hepatic/splenic HU < 1. Results. Among 200 patients, fatty liver was found in $67(33.5 \%)$ and nonfatty liver in 133 (66.5\%). Compared with patients without fatty liver, the severity of pancreatitis and levels of serum C-reactive protein were higher in fatty liver patients. The prevalence of local complications, persistent organ failure, and mortality were also higher in patients with fatty liver. Even after adjusting for age, sex, body mass index, and cause of pancreatitis, fatty liver was significantly associated with moderately severe or severe acute pancreatitis. Conclusions. Fatty liver may play a prognostic role in acute pancreatitis. Fatty liver could be incorporated into future predictive scoring models.
\end{abstract}

\section{Introduction}

Acute pancreatitis (AP) is an acute, painful abdominal disease involving the pancreas. Its incidence is on the rise, ranging from 13 to 45 per 100,000 [1]. AP is usually a mild disease, but approximately one-fifth of patients develop severe courses with a mortality rate of 10 to $20 \%$ [2-4]. It is important to categorize patients with different severity grades in order to prognosticate and triage, as severe cases require early intensive care and nutritional support. Over recent decades, several clinical, biochemical, and combined scoring models have been developed to assess the prognosis of AP $[5,6]$. However, each scoring system has certain shortcomings. For example, the Acute Physiology and Chronic Health Evaluation-II score is complex and the Ranson score is not measured until 48 hours after admission.
Serum C-reactive protein (CRP) level has been widely used as a simple prognostic biomarker, but the delay in peak levels makes it less useful on admission $[7,8]$.

In 2012, the Acute Pancreatitis Classification Working Group modified the Atlanta classification system to improve clinical assessment and treatment of AP [9]. The revised Atlanta classification system focuses mainly on morphologic manifestations by means of computed tomography (CT) [10]. A radiologic scoring system using CT was first introduced by Balthazar et al. in 1985, and a CT severity index with a 10 -point scale was developed later $[11,12]$. These radiologic scoring systems have been widely used in clinical practice, but they have only shown moderate interobserver agreement [13].

Fatty liver is commonly associated with benign gastrointestinal and pancreaticobiliary diseases, including acute 


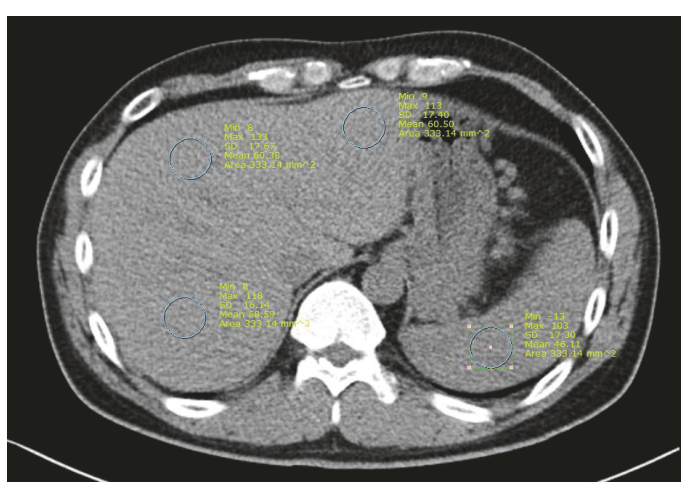

(a)

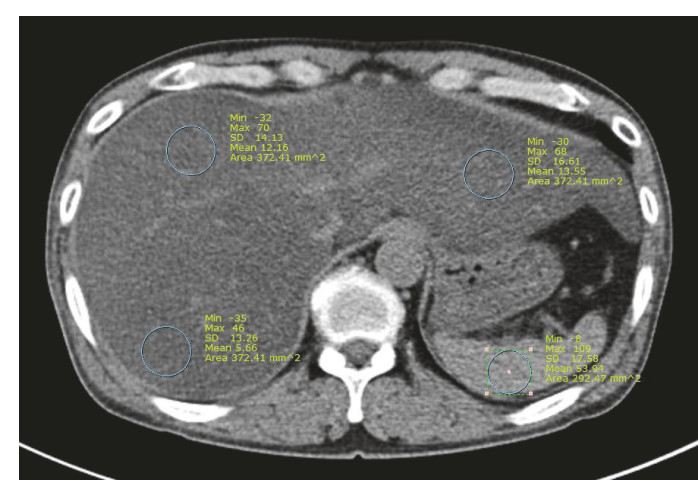

(b)

FIGURE 1: Unenhanced CT images from two patients with acute pancreatitis. (a) 54-year-old man with normal liver attenuation (60 HU) compared with spleen $(46 \mathrm{HU})$. (b) 46-year-old man with severe fatty liver. Mean liver attenuation (13 HU) was significantly lower than spleen attenuation $(54 \mathrm{HU})$.

pancreatitis [14]. Fatty liver change, which appears on CT as low attenuation, is frequently detected in AP patients; however, the influence of fatty liver on the severity and clinical outcomes of AP has not been well-studied. A previous study suggested that unenhanced CT demonstrated acceptable levels of sensitivity for detecting hepatic steatosis proved by biopsy [15]. CT scans are usually performed within 72 hours after admission to diagnose and stratify the severity of AP. Therefore, if the finding of fatty liver in an initial CT scan is associated with the severity of AP, it could be useful as an early detectable prognostic marker for in AP. The aim of this study was to investigate the influence of fatty liver on severity and outcomes of AP.

\section{Materials and Methods}

2.1. Subjects and Study Design. We retrospectively analyzed patients diagnosed with AP between July 2009 and June 2016 at Seoul St. Mary's Hospital in Seoul, Korea. Diagnosis criteria for AP were defined as the presence of at least 2 of the 3 following factors: (1) abdominal pain characteristic of AP, (2) serum amylase and/or lipase values of more than three times the upper limit of normal, and (3) characteristic findings of AP on CT scan [3]. For patients who were admitted to the hospital for AP more than once during the study period, only the first visit was included in the analysis. Exclusion criteria for the study were AP after endoscopic retrograde cholangiopancreatography, referred cases from other hospitals without an initial CT study, cases without CT scan or unenhanced CT phase, and missing body mass index (BMI) data. The institutional review board approved this study (KC16RISI0585).

2.2. Severity Assessment of Acute Pancreatitis. Based on the revised Atlanta classification, AP severity was stratified into three groups: mild, moderately severe, and severe [9]. Mild AP was classified as the absence of organ failure and the absence of local or systemic complications. Moderately severe AP was characterized by the presence of transient organ failure resolving within 48 hours and/or local or systemic complications without persistent organ failure. Severe AP was defined as persistent organ failure lasting more than 48 hours or death. Organ failure was defined as a score $\geq 2$ in one of the three assessed organ systems (cardiovascular, respiratory, and renal) using the modified Marshal scoring system [16]. Persistent organ failure was considered if organ failure lasted more than 48 hours. Local complications included acute peripancreatic fluid collection, pancreatic pseudocyst, acute necrotic collection, and walled-off necrosis. Systemic complications were defined as exacerbation of pre-existing comorbid disease, such as coronary artery disease of chronic lung disease, precipitated by the AP. Initial and maximum CRP levels during admission were investigated, and the length of hospital stay was assessed as an outcome parameter of AP.

2.3. Measurement of Fatty Liver. At our institution, an initial CT scan is usually performed in all AP patients within 72 hours after admission, regardless of AP severity. Unenhanced phase CT images from a picture archiving and communication system were retrospectively reviewed by a radiologist (M.H.C.) blinded to clinical and demographic data. The liver normally has a higher CT attenuation than the spleen. However, fatty liver change leads to a reversal of the liver-to-spleen attenuation ratio $[17,18]$. Two consecutive axial CT slices were used to measure the mean Housefield units (HU) for regions of interest (ROIs) in the liver and spleen. ROIs ranged from 200 to $400 \mathrm{~mm}^{2}$. In one image, two ROIs were placed in the right hepatic lobe, one ROI in the left hepatic lobe, and one ROI in the spleen (Figure 1). The mean hepatic HU was derived by averaging the HU of all three liver ROIs. The liver-to-spleen attenuation ratio was calculated by dividing the mean hepatic $\mathrm{HU}$ by splenic HU. Finally, the results from the two images were averaged. Fatty liver was defined as a liver-to-spleen attenuation ratio of less than 1 .

2.4. Statistical Analysis. Continuous data are presented as mean \pm SD or median (interquartile range), and categorical data are presented as quantities and proportions. Descriptive 
TABLE 1: Baseline characteristics and severity outcomes of study patients $(n=200)$.

\begin{tabular}{lc}
\hline Patient characteristics & \\
Age, mean \pm SD (range), years & $54.3 \pm 17.5(22-87)$ \\
Sex, male (\%) & $119(59.5 \%)$ \\
Etiology & $72(36.0 \%)$ \\
$\quad$ Gallstone-related & $67(33.5 \%)$ \\
$\quad$ Alcohol abuse & $39(19.5 \%)$ \\
Idiopathic & $22(11.0 \%)$ \\
$\quad$ Other & \\
Severity outcome & \\
Revised Atlanta classification & $110(55.0 \%)$ \\
$\quad$ Mild & $73(36.5 \%)$ \\
$\quad$ Moderately severe & $17(8.5 \%)$ \\
$\quad$ Severe & $68(34.0 \%)$ \\
Peripancreatic fluid collection & $57(28.5 \%)$ \\
Pancreatic pseudocyst & $28(14.0 \%)$ \\
Acute necrotic collection & $14(7.0 \%)$ \\
Walled-off necrosis & $14(7.0 \%)$ \\
Systemic complications & $17(8.5 \%)$ \\
Persistent organ failure & $3(1.5 \%)$ \\
Mortality & $7(5-11)$ \\
Duration of hospitalization, & \\
median (IQR), days & \\
\hline
\end{tabular}

IQR: interquartile range.

statistics were used to analyze the baseline characteristics of the study population. Comparisons of characteristics and variables between fatty liver and nonfatty liver groups were performed by using a two-sample independent $t$-test or the Mann-Whitney $U$ test for numerical variables and a Pearson chi-square test or the Fisher exact test for nominal variables. Factors potentially associated with moderately severe or severe AP were investigated using logistic regression analysis. The linear-by-linear association method was used for testing trends between fatty liver change and severity of AP in subgroup analyses. Statistical analyses were performed using SPSS 24.0 package (SPSS Inc., Chicago, IL, USA). Statistical significance was defined as $p<0.05$.

\section{Results}

3.1. Baseline Characteristics and Severity of Study Patients. A total of 285 patients were diagnosed with AP during the study period. Of these, 6 patients were transferred from an outside hospital without an early CT study. Two patients did not undergo initial CT scanning in our hospital and 72 patients did not undergo unenhanced phase CT. Five patients had missing data regarding BMI. After excluding these 85 patients, the remaining 200 patients were analyzed.

Baseline characteristics and severity outcomes of study patients $(n=200)$ are shown in Table 1 . The study included 119 males (59.5\%) and 81 females (40.5\%). The mean age was $54.3 \pm 17.5$ years. The cause of AP was gallstone-related in $72(36.0 \%)$ patients, alcohol abuse in $67(33.5 \%)$ patients, idiopathic in 39 (19.5\%) patients, and other in $22(11.0 \%)$ patients. Other causative factors were hypertriglyceridemia $(n=6)$, periampullary tumor $(n=6)$, drugs $(n=5)$, autoimmune-related $(n=3)$, and miscellaneous causes $(n=2)$. According to the revised Atlanta classification, 110 (55.0\%) cases were classified as mild, $73(36.5 \%)$ cases as moderately severe, and 17 (8.5\%) cases as severe. The incidences of acute peripancreatic fluid collection, pancreatic pseudocyst, acute necrotic collection, and walled-off necrosis were $68(34.0 \%), 57(28.5 \%), 28(14.0 \%)$, and $14(7.0 \%)$, respectively. Systemic complications and persistent organ failure occurred in $14(7.0 \%)$ and $17(8.5 \%)$ patients, respectively. There were $3(1.5 \%)$ cases of mortality during the study period, and the median (interquartile range) duration of hospitalization was 7 (5-11) days.

\subsection{Comparison of Characteristics and Severity in AP Patients} with and without Fatty Liver. Of the 200 enrolled patients, fatty liver was found in $67(33.5 \%)$ patients. Table 2 shows a comparison of the baseline characteristics and the severity parameters between patients with and without fatty liver. Mean age was not significantly different between fatty liver and nonfatty liver groups $(53.6 \pm 16.7$ versus $54.7 \pm 17.9$, $p=0.658)$. Fatty liver was more prevalent in male patients ( $76.1 \%$ versus $51.1 \%, p<0.001)$. Mean BMI and the rate of overweight or obese patients were higher in the fatty liver group compared with those in the nonfatty liver group $(25.3 \pm 4.6$ versus $23.4 \pm 3.3$ and $47.9 \%$ versus $25.2 \%$, all $p=0.001)$. Mean arterial pressure, waist circumference, and random glucose level were also higher in the fatty liver group. The prevalence of alcohol-induced pancreatitis was higher in the fatty liver group than that in the nonfatty liver group (44.8\% versus $27.8 \%, p=0.016)$. There were $19(28.4 \%)$ cases of mild AP, 38 (56.7\%) of moderately severe AP, and 10 of (14.9\%) severe AP in the fatty liver group, and 91 (68.4\%) cases of mild AP, 35 (26.3\%) of moderately severe AP, and $7(5.3 \%)$ of severe AP in the nonfatty liver group. The prevalence of mild AP was relatively lower, and the prevalence of moderately severe and severe AP were higher in the fatty liver group compared with that in the nonfatty liver group. Initial and maximum serum CRP levels [median (interquartile range)] of the fatty liver group were significantly higher than those of the nonfatty group [0.9 (0.2-9.6) versus $0.4(0.1-2.1), p=0.003$, and $15.6(4.1-24.5)$ versus $4.1(0.6-14.2), p<0.001$, resp.]. The median (interquartile range) duration of hospitalization was $8(6-13)$ and $7(5-10)$ days in fatty and nonfatty patients, respectively $(p=0.057)$.

3.3. Comparison of the Incidence of Complications in AP Patients with and without Fatty Liver. As shown in Figure 2, a significantly higher proportion of patients with fatty liver had various local or systemic complications compared to nonfatty liver patients. Patients with fatty liver showed higher percentages of acute peripancreatic fluid collection $(52.9 \%$ versus $24.1 \%, p<0.001)$ and acute necrotic collection $(20.9 \%$ versus $10.5 \%, p=0.046)$ than those without fatty liver. Patients with fatty liver also had higher 
TABLE 2: Comparison of characteristics and severity parameters between AP patients with and without fatty liver.

\begin{tabular}{|c|c|c|c|}
\hline Parameters & Fatty liver $(n=67)$ & Nonfatty liver $(n=133)$ & $p$ \\
\hline Age, mean $\pm S D$, years & $53.6 \pm 16.7$ & $54.7 \pm 17.9$ & 0.658 \\
\hline Sex, male (\%) & $51(76.1 \%)$ & $68(51.1 \%)$ & $<0.001$ \\
\hline Body mass index, $\mathrm{kg} / \mathrm{m}^{2}$ & $25.3 \pm 4.6$ & $23.4 \pm 3.3$ & 0.001 \\
\hline Overweight or obese (BMI $\left.\geq 25 \mathrm{~kg} / \mathrm{m}^{2}, \%\right)$ & $35(47.9 \%)$ & $32(25.2 \%)$ & 0.001 \\
\hline Mean arterial pressure, $\mathrm{mm} \mathrm{Hg}$ & $94.8 \pm 14.3$ & $90.7 \pm 12.7$ & 0.038 \\
\hline Waist circumference, $\mathrm{cm}$ & $90.7 \pm 10.0$ & $83.3 \pm 8.8$ & $<0.001$ \\
\hline Random blood glucose, mg/dL & $179.2 \pm 66.2$ & $150.4 \pm 71.0$ & 0.006 \\
\hline Serum triglyceride, $\mathrm{mg} / \mathrm{dL}$ & $224.9 \pm 312.4$ & $159.2 \pm 190.9$ & 0.123 \\
\hline Alcohol-induced pancreatitis (\%) & $30(44.8 \%)$ & $37(27.8 \%)$ & 0.016 \\
\hline Severity by revised Atlanta classification (\%) & & & $<0.001$ \\
\hline Mild & $19(28.4 \%)$ & $91(68.4 \%)$ & \\
\hline Moderately severe & $38(56.7 \%)$ & $35(26.3 \%)$ & \\
\hline Severe & $10(14.9 \%)$ & $7(5.3 \%)$ & \\
\hline Initial serum CRP level, median (IQR), mg/dL* & $0.9(0.2-9.6)$ & $0.4(0.1-2.1)$ & 0.003 \\
\hline Maximum serum CRP level, median (IQR), mg/dL* & $15.6(4.1-24.5)$ & $4.1(0.6-14.2)$ & $<0.001$ \\
\hline Duration of hospitalization, median (IQR), days & $8(6-13)$ & $7(5-10)$ & 0.057 \\
\hline
\end{tabular}

AP: acute pancreatitis; CRP: C-reactive protein; IQR: interquartile range; ${ }^{*}$ normal range of CRP: $0-0.3 \mathrm{mg} / \mathrm{dL}$.

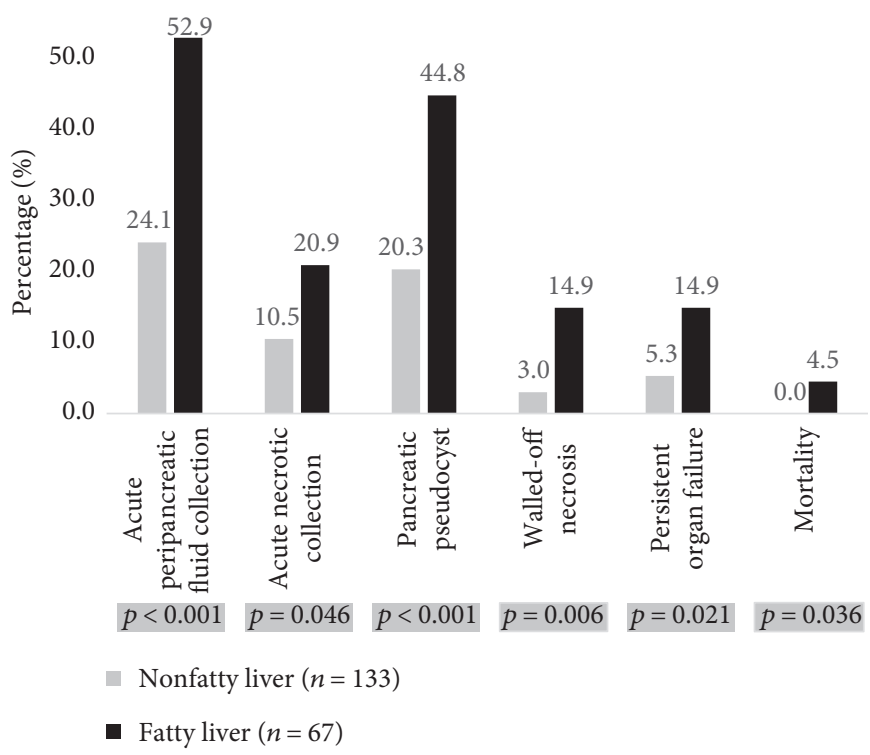

FIGURE 2: Comparison of complication rates of acute pancreatitis with and without fatty liver.

rates of chronic local complications including pancreatic pseudocyst $(44.8 \%$ versus $20.3 \%, p<0.001)$ and walled-off necrosis $(14.9 \%$ versus $3.0 \%, p=0.006)$. Persistent organ failure and mortality rates were also higher in fatty liver patients compared with those in nonfatty liver patients (14.9\% versus $5.3 \%, p=0.021$ and $4.5 \%$ versus $0.0 \%$, $p=0.036$, resp.).

3.4. Logistic Regression Analysis for Factors Associated With Moderately Severe or Severe AP. Univariate and multivariate logistic regression models for factors associated with moderately severe or severe AP are summarized in Table 3. Univariate analysis showed that fatty liver [odds ratio (OR), 5.47; 95\% confidence intervals (CI), 2.83-10.43] was a significant risk factor for moderately severe or severe AP. Even after adjusting for age, sex, BMI, and cause of pancreatitis, fatty liver was significantly associated with moderately severe or severe AP (OR, 4.95; 95\% CI, 2.46-9.98). Older age (OR, 1.03; 95\% CI, 1.01-1.05) and higher BMI (OR, 1.09; 95\% CI, 1.00-1.19) were also risk factors for moderately severe or severe AP. Sex and alcoholic cause were not significantly associated with the severity of AP in our analyses. 
TABLE 3: Logistic regression analysis for factors associated with moderately severe or severe acute pancreatitis.

\begin{tabular}{lcccc}
\hline \multirow{2}{*}{ Factor } & \multicolumn{2}{c}{$\begin{array}{c}\text { Univariate } \\
\text { analysis }\end{array}$} & \multicolumn{2}{c}{$\begin{array}{c}\text { Multivariate } \\
\text { analysis }\end{array}$} \\
& OR & $95 \%$ CI & OR & $95 \%$ CI \\
\hline $\begin{array}{l}\text { Fatty liver versus } \\
\text { nonfatty liver }\end{array}$ & $5.47^{*}$ & $2.87-10.43$ & $4.95^{*}$ & $2.46-9.98$ \\
Age & $1.02^{*}$ & $1.00-1.04$ & $1.03^{*}$ & $1.01-1.05$ \\
Male versus female & 1.34 & $0.76-2.37$ & 0.86 & $0.42-1.75$ \\
BMI & $1.11^{*}$ & $1.03-1.20$ & $1.09^{*}$ & $1.00-1.19$ \\
$\begin{array}{l}\text { Alcoholic cause versus } \\
\text { nonalcoholic cause }\end{array}$ & 1.55 & $0.86-2.80$ & 2.07 & $0.96-4.45$ \\
\hline
\end{tabular}

CI: confidence intervals; OR: odds ratio; $*$ indicates $p$ value of $<0.05$.

3.5. Subgroup Analyses. BMI and alcohol abuse are highly associated with fatty liver [19-21]. Therefore, subgroup analyses were done for the categories of BMI (BMI $\geq 25$ versus $\mathrm{BMI}<25$ ) and cause of pancreatitis (alcoholic versus nonalcoholic cause). Fatty liver was independently associated with severity of $\mathrm{AP}$, both in patients with $\mathrm{BMI} \geq 25$ and $\mathrm{BMI}<25$ ( $p$ for trend $=0.010$ and $<0.001$, resp.; Figure 3 ). Strong trends in fatty liver and severity of AP were also observed regardless of the cause of pancreatitis ( $p$ for trend $=0.017$ in alcoholic cause and $p$ for trend $<0.001$ in nonalcoholic cause, resp.).

\section{Discussion}

In this study, we evaluated the influence of fatty liver on severity and clinical outcome in AP. Our results showed that AP patients with fatty liver had more severe clinical features than patients without fatty liver. In patients with AP, fatty liver led to higher rates of local complications, persistent organ failure, and mortality. Fatty liver remained a significant risk factor for severe disease course even after adjusting for multiple confounding factors including age, BMI, and cause of AP.

Fatty liver disease (FLD) is a common hepatic metabolic disorder referring to a wide clinical spectrum ranging from simple hepatic steatosis to severe cirrhosis. According to etiology, fatty liver disease can be classified as alcoholic liver disease and nonalcoholic fatty disease. The prevalence of FLD is rapidly growing due to obesity and alcoholism epidemics, and it affects nearly one-fourth of the general population in both Western and Eastern countries [22, 23]. Beyond damage to the liver, fatty liver can induce diabetes, metabolic syndrome, and atherosclerosis [24-26]. Fatty liver at baseline can be related to higher health care utilization and costs of medical services. Thus, there is concern about FLD increasing [27].

Fatty liver is often seen in AP patients because both diseases share contributing factors such as obesity, alcohol abuse, and hyperlipidemia. In our study, about one-third of patients with AP demonstrated fatty liver change in nonenhanced CT images. Patients with fatty liver showed more severe features and poorer clinical outcomes compared to patients without fatty liver. Interestingly, fatty liver was significantly associated with AP severity regardless of subgroup analysis or adjustment for possible confounding factors, including age and BMI. These findings suggest that fatty liver by itself may be an independent risk factor for severe clinical course of AP. Fatty liver changes in early CT scan can be used as early prognostic markers and can be usefully incorporated into future predictive AP scoring models.

The mechanism for why fatty liver is associated with a more severe course of AP has not been elucidated. The elevation of serum CRP level in the fatty liver group may be one possible explanation for our results. Higher serum CRP levels were consistently found in fatty liver patients compared to controls in previous studies $[28,29]$. A chronic proinflammatory state in fatty liver patients may aggravate the course of AP. A recent study suggested that, in rat and human AP models, hepatic steatosis depressed alpha1antitrypsin levels, which have significant anti-inflammatory properties due to their effect on a wide range of inflammatory cells [30]. Decrease of serum alpha1-antitrypsin levels might lead to excessive activation of inflammation, and this proposed mechanism could support our results. In our study, the difference between initial CRP level in the fatty liver and nonfatty liver group was not great (median, 0.9 versus 0.4); however, the difference in maximum CRP between the two groups was pronounced (median, 15.6 versus 4.1). Serum CRP has been the most widely used biomarker, but the delay in peak levels makes it less useful on admission. Fatty liver in early CT scan can predict the delayed elevation of serum CRP; thus, fatty liver can be a prognostic marker easily determined at initial diagnosis.

In the present study, CT scan was used to assess fatty liver. A previous study suggested that liver and spleen attenuation from unenhanced CT could closely predict the degree of macrovesicular steatosis [15]. Many studies also used the liver-to-spleen attenuation ratio to define the fatty liver change $[17,18]$. CT scanning is a clinically suitable method for the assessment of fatty liver in AP patients, and contrast-enhanced CT is the primary tool used to assess and monitor AP. Moreover, the importance of CT has increased in patients with AP because the revised Atlanta classification system is based mainly on morphologic features. Combined unenhanced and enhanced CT scans may be useful in assessing the status of both fatty liver and AP.

On retrospective analysis, this study had some limitations. First, we acknowledge the limitation of excluding approximately one-fourth of patients who did not undergo unenhanced phase CT scan. The role of unenhanced CT imaging had not been established before our study, and the unenhanced phase has not been routinely performed in AP patients. Second, we could not exclude patients taking medications that could cause liver steatosis or could not consider the effects of such drugs. Third, the proportion of idiopathic AP in our study (19.5\%) was slightly higher than that generally known. Unfortunately, we could not evaluate genetic predisposition or sphincter of odd dysfunction as the cause of unexplained pancreatitis. In addition, endoscopic ultrasound or magnetic resonance cholangiopancreatography 


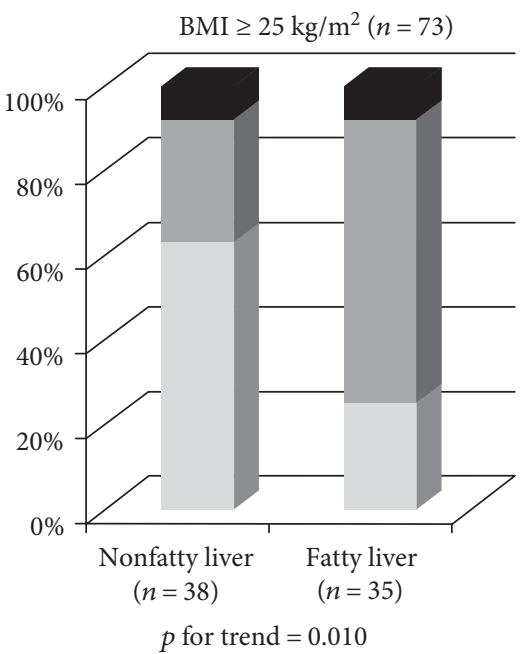

Severe

Mod. severe

Mild

(a)

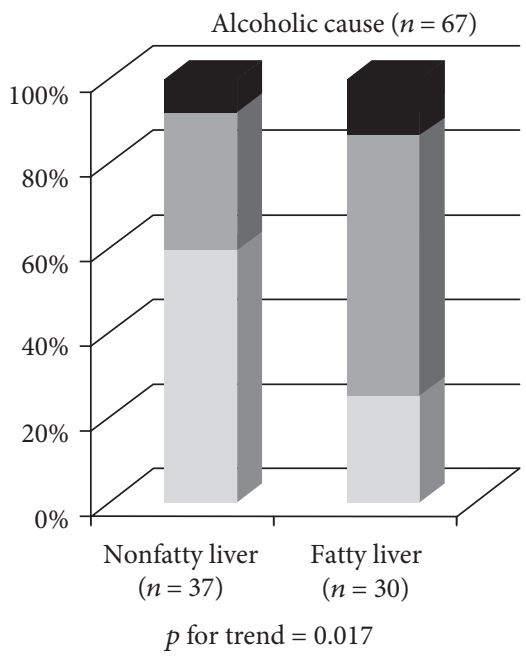

Severe

Mod. severe

Mild
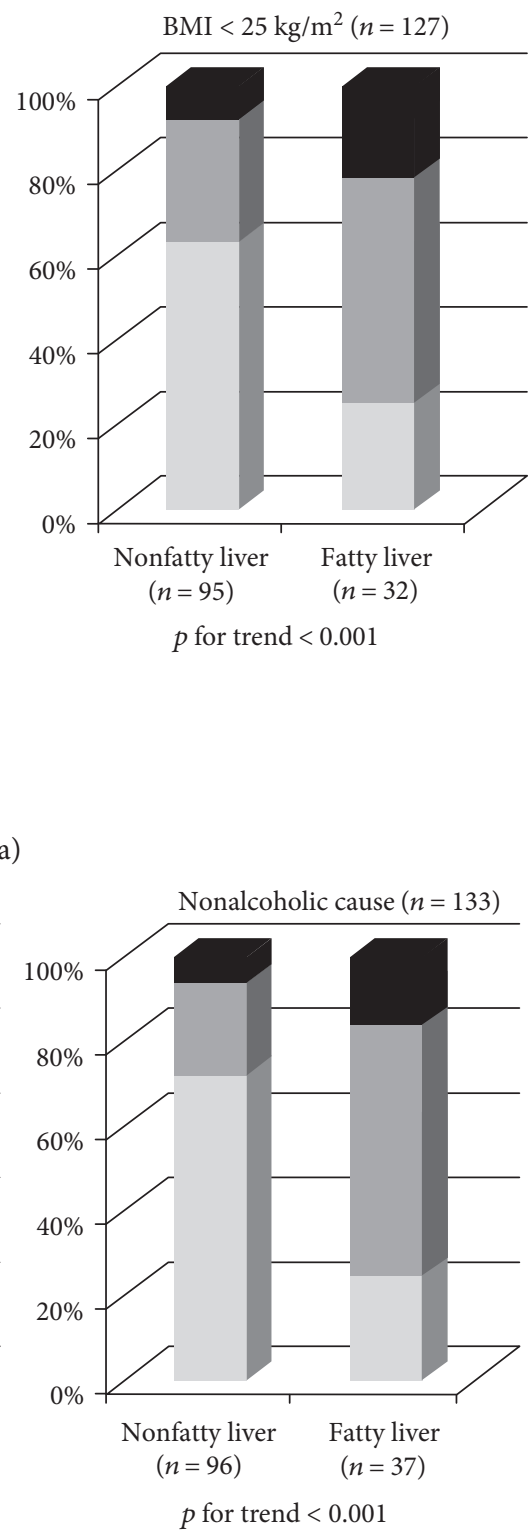

(b)

FIGURE 3: Subgroup analyses on the influence of fatty liver on clinical severity of acute pancreatitis (AP). (a) Fatty liver was associated with severity of $\mathrm{AP}$, in patients with $\mathrm{BMI} \geq 25$ and $\mathrm{BMI}<25$. (b) Fatty liver was also associated with severity of AP in patients with alcoholic cause of AP and nonalcoholic cause.

was not routinely performed in our patients with idiopathic AP. For these reasons, the rate of idiopathic AP would have been overestimated. Finally, pro- and anti-inflammatory cytokine levels were not examined, and the mechanism by which fatty liver aggravates AP remains to be defined. Further studies including cytokine data are needed to clarify the role of fatty liver in patients with AP.

In conclusion, fatty liver was frequently detected by unenhanced CT scan and was strongly correlated with AP severity. Fatty liver led to higher rates of local complications, persistent organ failure, and mortality in AP. This simple grading system is a potentially valuable prognostic marker that should be considered for use in future predictive scoring systems for AP.

\section{Conflicts of Interest}

There are no conflicts of interest. 


\section{Acknowledgments}

This work was supported by a grant from the National Research Foundation of Korea funded by the Korean Government (NRF-2015R1C1A1A02037568).

\section{References}

[1] D. Yadav and A. B. Lowenfels, "The epidemiology of pancreatitis and pancreatic cancer," Gastroenterology, vol. 144, no. 6, pp. 1252-1261, 2013.

[2] V. S. Swaroop, S. T. Chari, and J. E. Clain, "Severe acute pancreatitis," Jama, vol. 291, no. 23, pp. 2865-2868, 2004.

[3] P. A. Banks, M. L. Freeman, and G Practice Parameters Committee of the American College of, "Practice guidelines in acute pancreatitis," The American Journal of Gastroenterology, vol. 101, no. 10, pp. 2379-2400, 2006.

[4] D. C. Whitcomb, "Clinical practice. Acute pancreatitis," The New England Journal of Medicine, vol. 354, no. 20, pp. 2142-2150, 2006.

[5] M. Larvin and M. J. McMahon, "APACHE-II score for assessment and monitoring of acute pancreatitis," Lancet, vol. 2, no. 8656, pp. 201-205, 1989.

[6] J. H. Ranson and B. S. Pasternack, "Statistical methods for quantifying the severity of clinical acute pancreatitis," The Journal of Surgical Research, vol. 22, no. 2, pp. 79-91, 1977.

[7] I. A. Al Mofleh, "Severe acute pancreatitis: pathogenetic aspects and prognostic factors," World Journal of Gastroenterology, vol. 14, no. 5, pp. 675-684, 2008.

[8] C. J. Yang, J. Chen, A. R. Phillips, J. A. Windsor, and M. S. Petrov, "Predictors of severe and critical acute pancreatitis: a systematic review," Digestive and Liver Disease: Official Journal of the Italian Society of Gastroenterology and the Italian Association for the Study of the Liver, vol. 46, no. 5, pp. 446451, 2014.

[9] P. A. Banks, T. L. Bollen, C. Dervenis et al., "Classification of acute pancreatitis-2012: revision of the Atlanta classification and definitions by international consensus," Gut, vol. 62, no. 1, pp. 102-111, 2013.

[10] R. F. Thoeni, "The revised Atlanta classification of acute pancreatitis: its importance for the radiologist and its effect on treatment," Radiology, vol. 262, no. 3, pp. 751-764, 2012.

[11] E. J. Balthazar, J. H. Ranson, D. P. Naidich, A. J. Megibow, R. Caccavale, and M. M. Cooper, "Acute pancreatitis: prognostic value of CT,” Radiology, vol. 156, no. 3, pp. 767-772, 1985.

[12] K. J. Mortele, W. Wiesner, L. Intriere et al., "A modified CT severity index for evaluating acute pancreatitis: improved correlation with patient outcome," AJR American Journal of Roentgenology, vol. 183, no. 5, pp. 1261-1265, 2004.

[13] T. L. Bollen, V. K. Singh, R. Maurer et al., "A comparative evaluation of radiologic and clinical scoring systems in the early prediction of severity in acute pancreatitis," The American Journal of Gastroenterology, vol. 107, no. 4, pp. 612-619, 2012.

[14] S. K. Reddy, M. Zhan, H. R. Alexander, and S. S. El-Kamary, "Nonalcoholic fatty liver disease is associated with benign gastrointestinal disorders," World Journal of Gastroenterology, vol. 19, no. 45, pp. 8301-8311, 2013.

[15] P. Limanond, S. S. Raman, C. Lassman et al., "Macrovesicular hepatic steatosis in living related liver donors: correlation between CT and histologic findings," Radiology, vol. 230, no. 1, pp. 276-280, 2004.
[16] J. C. Marshall, D. J. Cook, N. V. Christou, G. R. Bernard, C. L. Sprung, and W. J. Sibbald, "Multiple organ dysfunction score: a reliable descriptor of a complex clinical outcome," Critical Care Medicine, vol. 23, no. 10, pp. 1638-1652, 1995.

[17] Y. Kodama, C. S. Ng, T. T. Wu et al., "Comparison of CT methods for determining the fat content of the liver," $A J R$ American Journal of Roentgenology, vol. 188, no. 5, pp. 13071312, 2007.

[18] C. J. Boyce, P. J. Pickhardt, D. H. Kim et al., "Hepatic steatosis (fatty liver disease) in asymptomatic adults identified by unenhanced low-dose CT," AJR American Journal of Roentgenology, vol. 194, no. 3, pp. 623-628, 2010.

[19] I. R. Wanless and J. S. Lentz, "Fatty liver hepatitis (steatohepatitis) and obesity: an autopsy study with analysis of risk factors," Hepatology, vol. 12, no. 5, pp. 1106-1110, 1990.

[20] M. R. Teli, C. P. Day, A. D. Burt, M. K. Bennett, and O. F. James, "Determinants of progression to cirrhosis or fibrosis in pure alcoholic fatty liver," Lancet, vol. 346, no. 8981, pp. 987-990, 1995.

[21] G. Vernon, A. Baranova, and Z. M. Younossi, "Systematic review: the epidemiology and natural history of non-alcoholic fatty liver disease and non-alcoholic steatohepatitis in adults," Alimentary Pharmacology \& Therapeutics, vol. 34, no. 3, pp. 274-285, 2011.

[22] P. Angulo, "Nonalcoholic fatty liver disease - reply," New England Journal of Medicine, vol. 347, no. 10, pp. 769-769, 2002.

[23] J. G. Fan, "Epidemiology of alcoholic and nonalcoholic fatty liver disease in China," Journal of Gastroenterology and Hepatology, vol. 28, Supplement 1, pp. 11-17, 2013.

[24] J. G. Fan and G. C. Farrell, "Epidemiology of non-alcoholic fatty liver disease in China," Journal of Hepatology, vol. 50, no. 1, pp. 204-210, 2009.

[25] H. X. Cao and J. G. Fan, "Editorial: Fatty liver disease: a growing public health problem worldwide," Journal of Digestive Diseases, vol. 12, no. 1, pp. 1-2, 2011.

[26] N. Chalasani, Z. Younossi, J. E. Lavine et al., "The diagnosis and management of non-alcoholic fatty liver disease: practice guideline by the American Association for the Study of Liver Diseases, American College of Gastroenterology, and the American Gastroenterological Association," Нераtology, vol. 55, no. 6, pp. 2005-2023, 2012.

[27] S. E. Baumeister, H. Völzke, P. Marschall et al., "Impact of fatty liver disease on health care utilization and costs in a general population: a 5-year observation," Gastroenterology, vol. 134, no. 1, pp. 85-94, 2008.

[28] N. Oruc, O. Ozutemiz, G. Yuce et al., "Serum procalcitonin and CRP levels in non-alcoholic fatty liver disease: a case control study," BMC Gastroenterology, vol. 9, no. 1, p. 16, 2009.

[29] M. Yoneda, H. Mawatari, K. Fujita et al., "High-sensitivity $\mathrm{C}$-reactive protein is an independent clinical feature of nonalcoholic steatohepatitis (NASH) and also of the severity of fibrosis in NASH," Journal of Gastroenterology, vol. 42, no. 7, pp. 573-582, 2007.

[30] Q. Wang, J. Du, P. Yu et al., "Hepatic steatosis depresses alpha-1-antitrypsin levels in human and rat acute pancreatitis," Scientific Reports, vol. 5, p. 17833, 2015. 


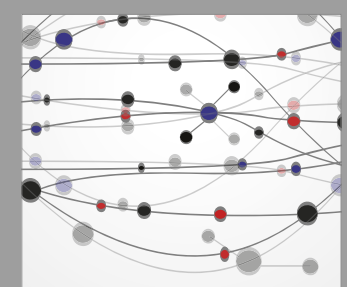

The Scientific World Journal
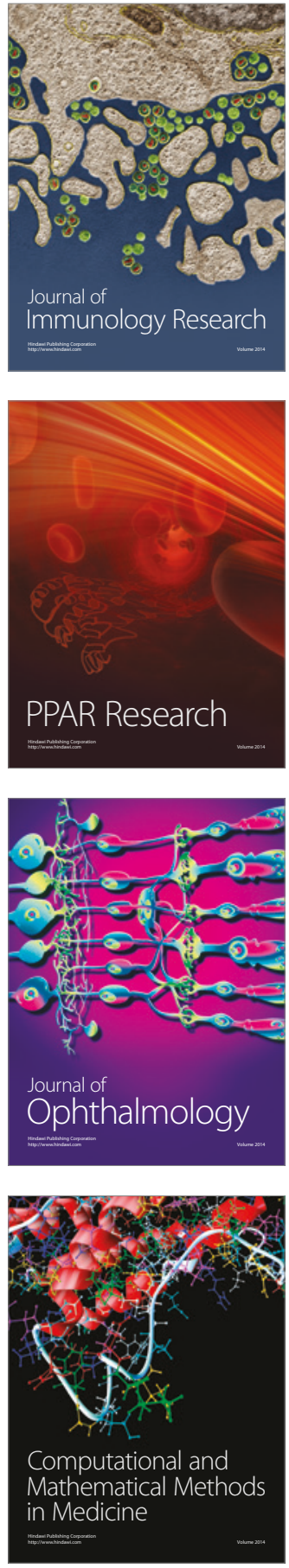

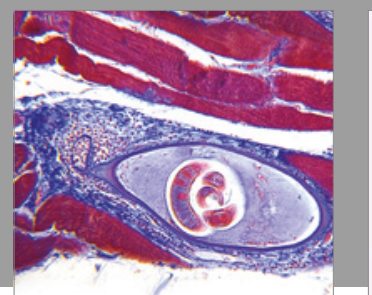

Gastroenterology Research and Practice
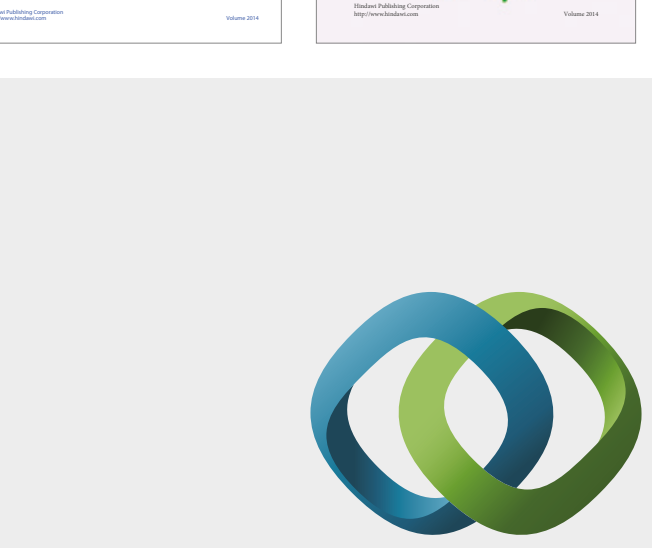

\section{Hindawi}

Submit your manuscripts at

https://www.hindawi.com
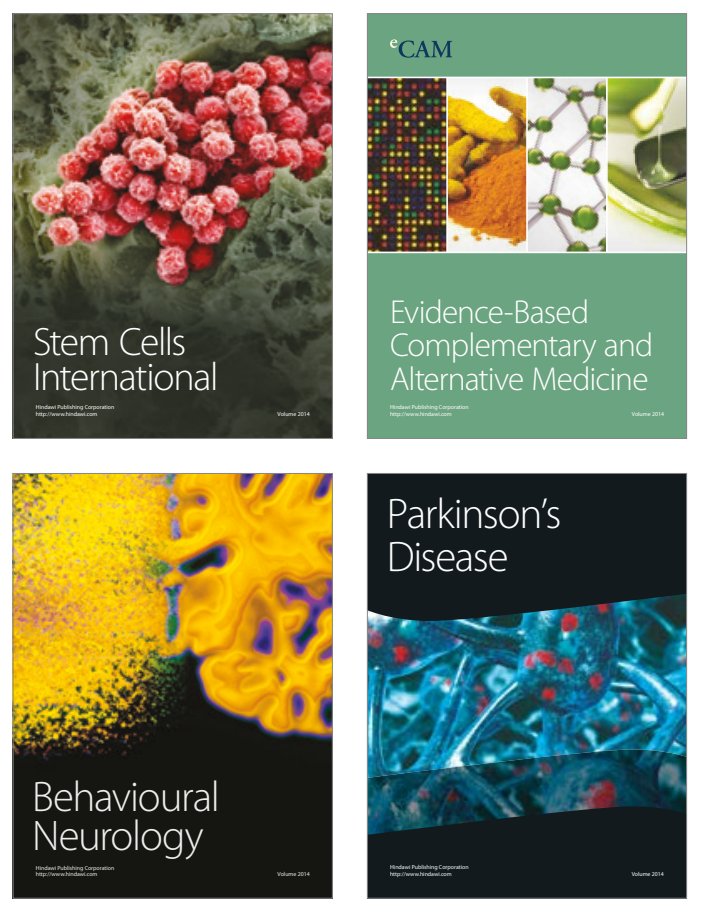
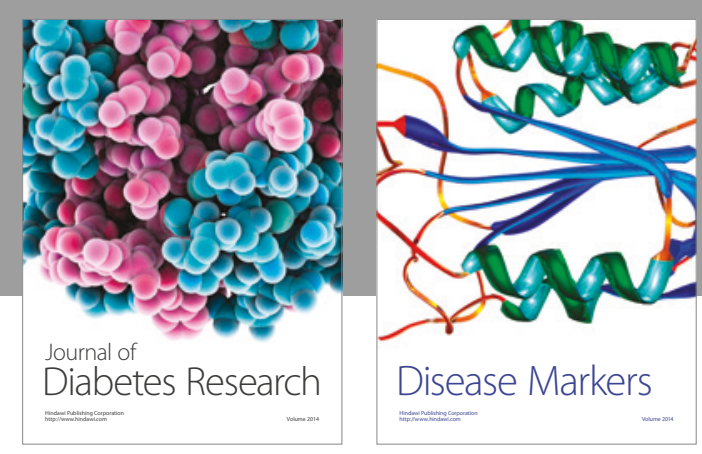

Disease Markers
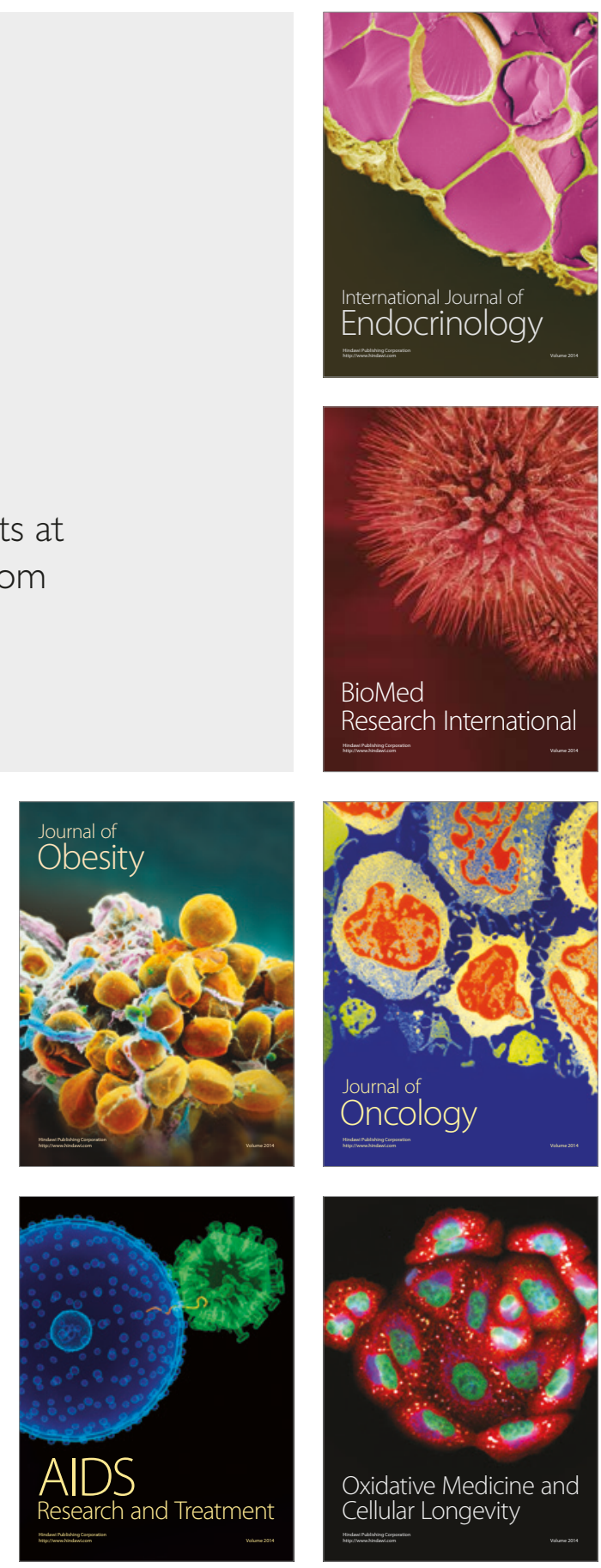